OPEN ACCESS

Edited by:

Tom Carr

Michigan State University,

United States

Reviewed by:

Miriam Gade,

Medical School Berlin, Germany

Giulia D'Aurizio,

Department of Applied Clinical

Sciences and Biotechnology,

University of L'Aquila, Italy

${ }^{*}$ Correspondence: Andrea Ballesio

andrea.ballesio@uniroma1.it

Specialty section:

This article was submitted to

Cognition,

a section of the journal

Frontiers in Psychology

Received: 10 February 2020

Accepted: 03 July 2020

Published: 05 August 2020

Citation:

Ballesio A, Cerolini S, Vacca M, Lucidi F and Lombardo C (2020) Insomnia Symptoms Moderate the Relationship Between Perseverative Cognition and Backward Inhibition

in the Task-Switching Paradigm.

Front. Psychol. 11:1837.

doi: 10.3389/fpsyg.2020.01837

\section{Insomnia Symptoms Moderate the Relationship Between Perseverative Cognition and Backward Inhibition in the Task-Switching Paradigm}

\author{
Andrea Ballesio ${ }^{1,2 *}$, Silvia Cerolini, ${ }^{1,2}$, Mariacarolina Vacca ${ }^{1}$, Fabio Lucidi $^{2}$ and \\ Caterina Lombardo ${ }^{1}$ \\ 1 Laboratory of Clinical Psychology and Psychophysiology, Department of Psychology, Sapienza University of Rome, Rome, \\ Italy, ${ }^{2}$ Department of Social and Developmental Psychology, Sapienza University of Rome, Rome, Italy
}

Perseverative cognition (PC), that is, the continuous cognitive representation of uncontrollable threats, is known to dampen executive control processes in experimental paradigms. Similarly, PC has been shown to impair sleep and to be implicated in the exacerbation of insomnia, which may in turn contribute to the disruption of executive functions. The interactions between PC and insomnia in influencing executive functions, however, have never been tested to date. In the present study, we explored whether insomnia symptoms may moderate the associations between PC and disrupted executive functions, with the hypothesis to find a stronger relationship between these variables at increasing levels of insomnia. Fifty participants completed measures of trait $\mathrm{PC}$ and insomnia severity in the previous month and also completed a computerized task-switching paradigm assessing backward inhibition, switch cost, and accuracy. Prior to the task switching, participants completed a measure of state rumination in order to control for the effects of state PC on cognitive performance. Results show that trait PC was significantly correlated with higher insomnia symptoms and state rumination and marginally correlated with lower backward inhibition and longer switch cost. Moreover, insomnia severity moderated the relationship between trait PC and backward inhibition after controlling for the effects of state rumination; that is, the relationship between PC and inhibitory deficits was stronger in those with higher versus lower levels of insomnia symptoms. Findings suggest the need to better elucidate the associations between PC, insomnia, and executive functioning in clinical samples and longitudinal designs.

Keywords: perseverative cognition, insomnia, task switching, inhibition, flexibility

\section{INTRODUCTION}

Perseverative cognition (PC), also referred to as repetitive negative thinking (e.g., Ehring et al., 2011; Law and Tucker, 2018) or perseverative negative thinking (e.g., Trick et al., 2016), is defined as the repeated or chronic activation of the cognitive representation of one or more psychological stressors. Worry and rumination are often recognized as the two main manifestations of PC. Worry is traditionally defined as a chain of negatively affect-laden thoughts and images related to 
relatively uncontrollable future outcomes, and it is traditionally associated with generalized anxiety (Borkovec et al., 1983). Rumination is conceptualized as repetitive thoughts about the origins and consequences of one's personal concerns or negative mood, and it is traditionally studied in the context of depression (Nolen-Hoeksema et al., 2008). Although different in their content, rumination and worry share common underlying cognitive and emotional processes, including intrusiveness, repetitiveness and uncontrollability of the thought, and negative affectivity (Ehring et al., 2011). Moreover, PC has been associated with negative health outcomes. For instance, metaanalytic evidence shows that PC is associated with alterations in cardiovascular, autonomic, and endocrine systems activity (Ottaviani et al., 2016). Additionally, PC tendency is considered a risk factor for developing mental disorders, including depression (Raes, 2012), anxiety (Ruscio et al., 2011), drinking behavior (Caselli et al., 2010), psychosis (Hartley et al., 2017), and to be involved in suicide ideation and behavior (Law and Tucker, 2018). Furthermore, because the experience of PC involves the difficulty to inhibit negative stimuli (e.g., a negative thought) or to shift attention away from them, PC has been associated with deficits in executive functions, and especially with the performance in cognitive tasks requiring attention switching and inhibitory abilities (see Yang et al. (2016) for a meta-analysis). For instance, high trait perseverators are shown to perform poorer on switching tasks compared to nonperseverators (e.g., Beckwé et al., 2014). Moreover, experimental and longitudinal findings confirmed that the induction of PC is associated with performance lapses in tasks requiring switching and inhibitory abilities (Whitmer and Gotlib, 2012; Connolly et al., 2014). Also, PC has been associated with disturbed sleep and insomnia symptoms (i.e., difficulties falling asleep and/or maintaining sleep) in ecological momentary assessments (Lancee et al., 2017) and laboratory (Zoccola et al., 2009; Ballesio et al., 2020) studies. Interestingly, insomnia has been associated with poor executive functions. Insomnia is the most common sleep disorder, with epidemiological studies estimating the prevalence of the insomnia diagnosis up to $20 \%$ in Europe (Chaput et al., 2018). Insomnia is defined by difficulties falling asleep and/or maintaining sleep accompanied by significant impairment in daytime functioning, such as fatigue, emotion dysregulation, and cognitive impairment (American Psychiatric Association, 2013). Importantly, insomnia is highly prevalent among those with mental disorders (American Psychiatric Association, 2013), and recent meta-analysis highlighted that insomnia is a risk factor for the development of depression, anxiety, alcohol use, and psychotic disorders (Hertenstein et al., 2019). With respect to cognitive impairment of insomnia, a recent metaanalysis comparing the cognitive performance of individuals with insomnia and good sleepers showed that insomnia is associated with moderate inhibitory $(d=-0.32)$ and switching $(d=-0.30)$ deficits (Ballesio et al., 2019). Therefore, it is reasonable to hypothesize that PC may interact with insomnia in influencing inhibitory and switching capacities; that is, the effects of PC on inhibitory and switching capacities may be stronger in those reporting insomnia symptoms compared to those without sleep problems. Notably, previous research on PC and executive functions has focused more on the content of thoughts than on the underlying cognitive processes. For example, in their study, Whitmer and Banich (2007) found that depressive rumination was associated with a deficit in the inhibition of previously relevant information, whereas angry rumination was associated with impairments in the ability to switching to a new task. In last years, however, it became clear that PC is a transdiagnostic factor associated with many psychological disorders (Wahl et al., 2019); namely, the cognitive processes underlying PC are essentially the same across disorder-specific content of cognitions (Ehring et al., 2011). Therefore, more recent research is moving toward the study of the process of PC rather than the content, using content-independent measures of PC. However, research relating the process of PC to executive functions and examining the role of insomnia symptoms is still lacking. Therefore, the aim of this study was to test the effects of PC, as assessed using a contentindependent measure and insomnia symptoms on the taskswitching paradigm, a widely used experimental task assessing inhibitory and noninhibitory switching capacities (Ballesio et al., 2018a) in a sample of university students.

\section{MATERIALS AND METHODS}

\section{Participants}

A total sample of 50 participants were enrolled in the study. Participants were unselected university students recruited from the university community of the Faculty of Medicine and Psychology of Sapienza University of Rome through flyers, word of mouth, and announcement during lectures. Participants received no compensation to participate in the study and completed an informed consent prior to the beginning of the study.

\section{Measures}

\section{Perseverative Thinking Questionnaire}

The Perseverative Thinking Questionnaire (PTQ; Ehring et al., 2011) was used to assess trait PC. The PTQ is a 15-item content-independent measure of PC. It captures cognitive processes underlying PC, including core characteristics of PC (repetitiveness, intrusiveness, and difficulties with disengagement from the thought), perceived unproductiveness of the thought, and mental capacity engaged during PC. Items of the PTQ include "The same thoughts keep going through my mind again and again," "Thoughts intrude into my mind," "My thoughts take up all my attention." In our sample, the PTQ had high reliability $(\alpha=0.95)$.

\section{Insomnia Severity Index}

The Insomnia Severity Index (ISI; Bastien et al., 2001) was used to assess the presence and severity of insomnia symptoms (for instance, difficulties falling asleep and/or maintaining sleep, nonrestorative sleep, impact of insomnia on daytime functioning). Insomnia Severity Index score ranges from 0 (insomnia absent) to 28 (very severe insomnia), with 7 as the cutoff for subclinical insomnia and 14 the cutoff for identifying individuals with clinical insomnia in clinical samples. 
In community samples, a cutoff of 10 is usually suggested to detect individuals with high versus low insomnia symptoms, due to the high sensitivity $(86.1 \%)$ and specificity $(87.7 \%)$ of this cutoff in detecting insomnia cases (Morin et al., 2011). In our sample, the ISI had good reliability $(\alpha=0.76)$.

\section{Brief State Rumination Inventory}

The Brief State Rumination Questionnaire (BSRI; Marchetti et al., 2019) is a short (eight items) self-reported scale assessing state rumination. In absence of a content-independent measure of state PC, the BSRI was used to control for the effects of state PC on task-switching performance. This was done in line with experimental studies showing that the induction of rumination determines an impairment in task-switching processes (Whitmer and Gotlib, 2012). The BSRI was administered right before taskswitching implementation. Items of the BSRI include "Right now, I dwell on negative aspects of myself that I wish I'd stop thinking about," "Right now, it is hard for me to shut off negative thoughts about myself," "Right now, I dwell on negative aspects of myself that I wish I'd stop thinking about." In our sample, the BSRI had high reliability $(\alpha=0.90)$.

\section{Task-Switching Paradigm}

The task-switching paradigm (Figure 1) was used to assess backward inhibition and switch cost. We followed the same procedure described in detail in Ballesio et al. (2018a,b, 2020). The task is composed of three different rules that need to be applied in random sequences. The concept of backward inhibition involves that switching back to a recently executed task is harder than switching back to a less recently executed task due to residual inhibition. The backward inhibition index reflects slower reaction times (RTs) on the third trial of alternating triplets (A-B-A) versus the third trial of nonalternating triplets

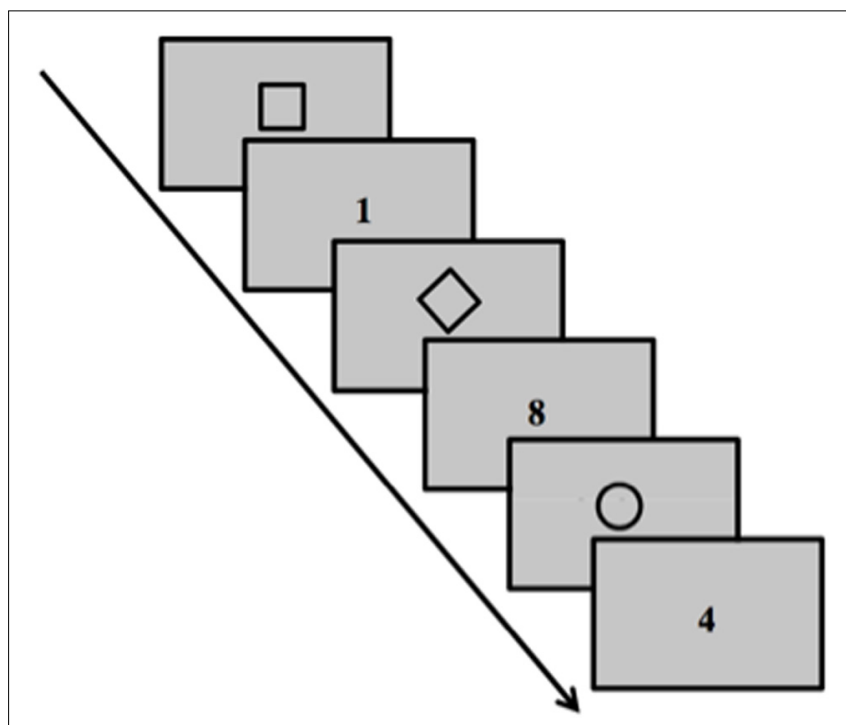

FIGURE 1 | Task Switching paradigm. Schematic representation of task stimuli. The task was composed of 162 trials presented on a 19" computer monitor placed frontally at a distance of about $70 \mathrm{~cm}$. sequences (C-B-A) that is due to residual inhibition suffered by rule $\mathrm{A}$ in $\mathrm{A}-\mathrm{B}-\mathrm{A}$ versus $\mathrm{C}-\mathrm{B}-\mathrm{A}$ sequences. Moreover, switching from one rule to another implies performance costs, that is, the switch cost. This is reflected in the increase in RTs in switching trials (A-B-A, C-B-A) compared to repetition trials (A-A-A).

\section{Procedure}

Recruited participants completed an informed consent sheet and self-reported questionnaires during an individual laboratory session. Questionnaires included PTQ and ISI and other measures of sleep and cognitive aspects of insomnia disorder not reported in the present study. Following in-laboratory assessment with psychological questionnaires, participants completed the task-switching paradigm assessing executive functions. Right before starting the task, participants completed the BSRI in order to control the presence of state PC. The procedure was approved by institutional review board of the Department of Psychology, Sapienza University of Rome.

\section{Data Preparation and Analysis}

Mean RTs for each sequence (repetition, alternating, switching) of the task switching were collected and computed using SuperLab (Cedrus Corporation, Inc.). Trial errors were not included in the calculations of backward inhibition and switch cost indices. In addition, RTs of \pm 2 standard deviations from the mean were not included in the calculations. Statistical analysis was computed using IBM SPSS 24. Data were checked for normal distribution (substantial departure from normality was considered as skew value $>2$ and kurtosis value $>7$ ). Descriptive analysis of the sample was performed. One-way analysis of variance (ANOVA) was performed to assess the differences in RTs associated with AAA, ABA, and CBA sequences of the task switching. Furthermore, mixeddesign factorial ANOVAs were implemented to examine the interactions between the within-factor task-switching RTs and the between-factor of the group (insomnia vs. good sleepers). Bivariate correlations between the main study variables (PTQ, BSRI, ISI, backward inhibition, switch cost, accuracy) were performed. Furthermore, to estimate the effects of PTQ on task-switching paradigm at different insomnia levels, the sample was split according to ISI scores (Morin et al., 2011) in high insomnia symptoms (ISI > 10) or low insomnia symptoms (ISI $<10$ ), and partial correlations were computed in the two subsamples between PTQ and taskswitching indices controlling for the effects of BSRI. Finally, conditional process modeling (Hayes, 2013) was performed to assess the hypothesis that insomnia severity may moderate the relationship between PC and executive functions. In these analyses, PTQ was inserted as independent variable, ISI was inserted as moderator, and task-switching indices were inserted as outcomes. Moreover, to control for the effects of state rumination on task switching, BSRI was inserted as covariate. Two separate analyses were computed for each task-switching outcome (backward inhibition, switch cost). According to our main hypothesis, we expected to find significant PTQ $\times$ ISI interactions on backward inhibition and switch cost. 
Moderation analysis was computed on PROCESS macro for SPSS (Hayes, 2013).

\section{RESULTS}

\section{Sample Characteristics}

Participants were on average $21.85 \pm 5.62$ years old. Females included in the study were $66 \%$. Participants were all Caucasian. Participants answered correctly at $50.78 \pm 5.11$ trial on 54 (94.03\%). Mean RTs were $715.55 \pm 216.09$ in the repetition sequences (AAA), $843.02 \pm 302.65$ in alternating sequences (ABA), and 878.24 \pm 292.21 in switching sequences (CBA). Backward inhibition score was on average $-30.36 \pm 154.87$, and switch costs score was $145.08 \pm 134.23$. Moreover, participants scored on average $7.65 \pm 4.27$ on ISI, $24.72 \pm 13.60$ on PTQ, and $215.49 \pm 164.46$ on BSRI.

\section{Manipulation Check}

One-way ANOVA was performed to assess the differences in RTs associated with AAA, ABA, and CBA sequences of the task switching. Pairwise comparisons showed that AAA sequences were significantly slower with respect to both ABA and CBA sequences (both $p<0.01$ ). Reaction times in ABA and CBA were not significantly different $(p=0.147)$. Mixed-design factorial ANOVA examining the interactions between task-switching sequences (RTs in AAA vs. ABA vs. CBA) $\times$ group (insomnia vs. good sleep) showed no significant interactions between the two variables $(F=1.980, \mathrm{df}=2, p=0.150)$.

\section{Correlations}

Results on entire sample showed that PC (PTQ) was significantly associated with insomnia severity (ISI, $r=0.41, p<0.01$ ) and state rumination (BSRI, $r=0.39, p<0.01$ ). Small and marginally significant associations were found between PTQ and backward inhibition $(r=-0.18, p=0.10)$ and switch cost $(r=0.21$, $p=0.07)$. PTQ did not correlate with task-switching accuracy $(r=-0.08, p=0.28)$.

To estimate the effects of PTQ on task-switching paradigm at different insomnia levels, the sample was split according to ISI scores in high insomnia symptoms $(n=18)$ or low insomnia symptoms (ISI $=32$ ), and partial correlations were computed in the two subsamples between PTQ and task-switching indices controlling for the effects of BSRI. Results in the group of high insomnia showed that PTQ was significantly and highly correlated with backward inhibition $(r=-0.57, p=0.01)$ and with switch cost $(r=0.44, p=0.050)$ but not with taskswitching accuracy $(r=-0.16, p=0.28)$. Contrarily, in the group of low insomnia, PTQ was not correlated with taskswitching performance (backward inhibition: $r=0.17, p=0.17$; switch cost: $r=0.11, p=0.28$; accuracy: $r=0.07, p=0.36)$.

\section{Moderation Analysis}

A first moderation analysis was performed with PTQ as independent variable, ISI as moderator, and backward inhibition as outcome. In the analysis, BSRI scores were

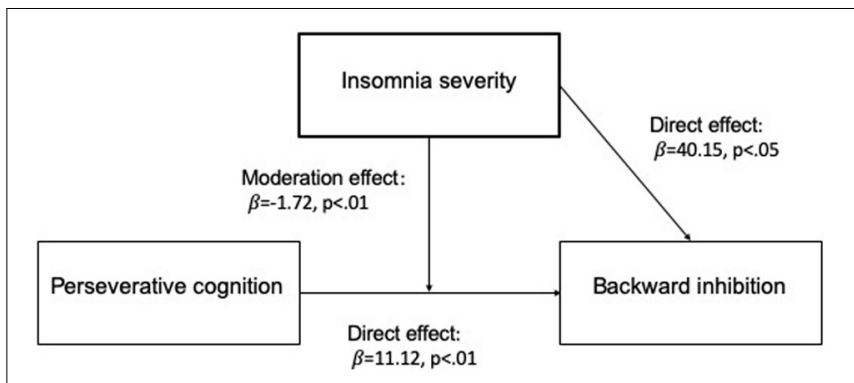

FIGURE 2 | Graphical representation of the moderation model.

inserted as covariate to control for the effects of state PC on task-switching performance. Results (Figure 2) show that the model was significant $\left(F_{(4,39)}=2.61, p<0.05\right)$. Backward inhibition was significantly predicted by PTQ $(\beta=11.12$, $p<0.05)$ and ISI $(\beta=40.15, p<0.05)$. Moreover, backward inhibition was predicted by the interaction PTQ $\times$ ISI $(\beta=-1.72, p<0.01)$, with a significantly increased $R^{2}$ due to interaction $\left(R\right.$ change $=0.18, F_{(1,39)}=8.72$, $p<0.05)$. The effect of BSRI on backward inhibition was not significant $(\beta=0.06, p>0.05)$.

A second model was computed with PTQ as independent variable, ISI as moderator, BSRI as covariate, and switch cost as outcome. Results show that the model was not significant $\left(F_{(4,39)}=0.87, p>0.05\right)$. Specifically, neither PTQ $(\beta=-1.88$, $p>0.05)$, nor ISI $(\beta=-12.71, p>0.05)$, nor BSRI $(\beta=0.05$, $p>0.05)$, nor PTQ $\times$ ISI interaction $(\beta=-53, p>0.05)$ significantly predicted switch cost. Because the correlation between PTQ and task-switching accuracy was below 0.10 (Cohen, 1992), we did not compute a moderation analysis with accuracy as outcome.

\section{DISCUSSION}

The aim of this study was to test the hypothesis that insomnia symptoms may moderate the relationship between PC and executive functions as assessed using a task-switching paradigm. This paradigm was chosen as a valid tool to study switch cost and backward inhibition in healthy and clinical populations, as documented by several papers from our group (Ballesio et al., 2018a,b, 2020; Cerolini et al., 2020) and other groups (e.g., Sdoia and Ferlazzo, 2008; Couyoumdjian et al., 2010). However, in the present study, we found that while RTs were significantly slower in alternating $(\mathrm{ABA})$ and switching $(\mathrm{CBA})$ when compared to repetition (AAA) trials, reflecting the switch cost, the difference between RTs in ABA and CBA trials was not significant. We explored whether this lack of backward inhibition effect could be due to the clinical condition of the included sample, but mixed-models analysis showed absence of a task $\times$ group interaction on RTs. This result is consistent with the findings reported by Philipp and Koch (2006), in which size of $N-2$ repetition cost (backward inhibition effect) is affected by the occurrence of task repetitions. Taken together, the inconsistencies in the results yielded from studies using this paradigm may 
reflect the influence on several variables influencing the presence and magnitude of the backward inhibition. However, it was beyond the aim of our study to assess the appropriateness of the task-switching paradigm to detect backward inhibition. Results of the correlations and the moderation models partially support the view that task-switching performance may be influenced by repetitive thought and insomnia. We found that PC is significantly associated with backward inhibition and switch cost in individuals reporting symptoms of insomnia but not in individuals reporting good sleep. Moreover, we found that insomnia moderates the relationship between PC and the performance in the backward inhibition, but not in the switch cost. Findings on backward inhibition are consistent and expand previous literature showing detrimental effects of PC (e.g., rumination, Hilt et al., 2014) and insomnia (e.g., Ballesio et al., 2019) on inhibitory capacities. Moreover, for the first time, here we show that PC and insomnia interact in influencing inhibitory control processes involved in the task switching. Specifically, results show that the negative effects of PC on inhibitory capacities increase at increasing levels of insomnia symptoms.

Results also show that neither PC (neither assessed with a trait nor with a state measure) nor insomnia was associated with switch cost in moderation analysis. This result is in contrast with a recent meta-analysis finding a positive correlation between PC (rumination) and switching capacities (Yang et al., 2016). However, this is consistent with a previous experimental study using the task-switching paradigm and showing that inducing rumination impacts switch cost in individuals with major depression but not in healthy controls (Whitmer and Gotlib, 2012). This is also consistent with a previous study showing that depressive rumination is associated with backward inhibition but not with noninhibitory switching abilities (Whitmer and Banich, 2007). Also, it must be noted that, in contrast with previous literature, we studied PC using a content-independent measure. Specifically, the cognitive correlates of the PTQ have never been investigated to date. It is therefore possible that this specific instrument may have limited sensitivity in detecting noninhibitory switching abilities.

\section{Clinical Implications}

Results from the present study may suggest several clinical implications. Both PC and insomnia are considered as transdiagnostic factors associated with several psychopathological conditions including mood and anxiety disorders, obsessive-compulsive disorders, posttraumatic stress disorders, and personality disorders and psychosis (Harvey, 2008; Wahl et al., 2019). Importantly, meta-analyses show that these disorders are associated with moderate-large inhibitory control impairments (Snyder et al., 2015). Therefore, if confirmed with longitudinal or experimental studies in clinical populations, our findings would highlight the importance to target both PC and insomnia in clinical interventions in order to improve cognitive functioning. Preliminary evidence, for instance, suggests that behavioral treatment of insomnia has a moderate impact on PC (e.g., Ballesio et al., 2018b; van der Zeerde et al., 2019).
Similarly, clinical protocols for reducing form of PC, such as cognitive behavioral therapy for worry, are associated with a reduction in insomnia symptoms (Bush et al., 2012). However, randomized controlled trials exploring the mediation effects of insomnia and PC on cognitive functioning are lacking and therefore encouraged.

\section{Limitations}

Several limitations need to be acknowledged. As the first study exploring the combined effect of PC and insomnia on task switching, we adopted a cross-sectional design. However, to infer stronger causal relationships between variables under study, future research is strongly recommended to employ experimental and longitudinal designs. Furthermore, we assessed $n-1$ switch and $n-2$ repetition cost in a single task-switching session, which may reduce the involvement of inhibitory processes (Hartmann et al., 2019). Moreover, our study comprised a convenience and small sample of university students. Notably, university students are particularly at risk of suffering from insomnia due to stress and academic demands and dysfunctional sleep habits (e.g., late-night computer work, substance use, irregular bed timing) (Jiang et al., 2015). Specifically, a recent metaanalysis of epidemiological literature comprising 16,478 participants estimated the prevalence of insomnia at $18.5 \%$ of university students. However, our sample was unselected, and therefore future larger studies are needed to replicate our findings in clinical samples in order to increase the generalizability of our findings.

\section{DATA AVAILABILITY STATEMENT}

The datasets generated for this study are available on request to the corresponding author.

\section{ETHICS STATEMENT}

The studies involving human participants were reviewed and approved by Institutional Review Board Department of Psychology, Sapienza University of Rome. The patients/participants provided their written informed consent to participate in this study.

\section{AUTHOR CONTRIBUTIONS}

$\mathrm{AB}$ planned the experiment, ran the analysis, and wrote the manuscript. MV and SC helped with the data analysis. FL and CL revised the final draft of the manuscript. All authors contributed to the article and approved the submitted version.

\section{ACKNOWLEDGMENTS}

We thank Ms. Michela Forcella for the help with the data collection. 


\section{REFERENCES}

American Psychiatric Association (2013). Diagnostic and Statistical Manual of Mental Disorders: DSM5. Washington, DC: American Psychiatric Association.

Ballesio, A., Aquino, M. R. J., Ferlazzo, F., Kyle, S. D., and Lombardo, C. (2019). Executive functions in insomnia disorder: a systematic review and exploratory meta-analysis. Front. Psychol. 10:101. doi: 10.3389/fpsyg.2019. 00101

Ballesio, A., Cerolini, S., Ferlazzo, F., Cellini, N., and Lombardo, C. (2018a). The effects of one night of partial sleep deprivation on executive functions in individuals reporting chronic insomnia and good sleepers. J. Behav. Ther. Exp. Psychiatry. 60, 42-45. doi: 10.1016/j.jbtep.2018. 02.002

Ballesio, A., Devoto, A., and Lombardo, C. (2018b). Cognitive behavioural therapy for insomnia reduces ruminative thinking. Sleep Biol. Rhythms. 16, 371-372. doi: 10.1007/s41105-018-0168-4

Ballesio, A., Ghezzi, V., Vacca, M., Ottaviani, C., and Lombardo, C. (2020). Effects of presleep cognitive intrusions on subjective sleep and next-day cognitive performance in insomnia. Behav. Ther. (in press). doi: 10.1016/j.beth.2019. 09.003

Bastien, C. H., Vallières, A., and Morin, C. M. (2001). Validation of the insomnia severity index as an outcome measure for insomnia research. Sleep Med. 2, 297-307. doi: 10.1016/s1389-9457(00)00065-4

Beckwé, M., Deroost, N., Koster, E. H. W., Lissnyder, E. D., and De Raedt, R. (2014). Worrying and rumination are both associated with reduced cognitive control. Psychol. Res. 78, 651-660. doi: 10.1007/s00426-013-0517-5

Borkovec, T. D., Robinson, E., Pruzinsky, T., and DePree, J. A. (1983). Preliminary exploration of worry: some characteristics and processes. Behav. Res. Ther. 21, 9-16. doi: 10.1016/0005-7967(83)90121-3

Bush, A. L., Armento, M. E. A., Weiss, B. J., Rhoades, H. M., Novy, D. M., Wilson, N. L., et al. (2012). The pittsburgh sleep quality index in older primary care patients with generalized anxiety disorder: psychometrics and outcomes following cognitive behavioral therapy. Psychiatry Res. 199, 24-30. doi: 10.1016/ j.psychres.2012.03.045

Caselli, G., Ferretti, C., Leoni, M., Rebecchi, D., Rovetto, F., and Spada, M. M. (2010). Rumination as a predictor of drinking behaviour in alcohol abusers: a prospective study. Addiction 105, 1041-1048. doi: 10.1111/j.1360-0443.2010. 02912.x

Cerolini, S., Ballesio, A., Lucidi, F., and Lombardo, C. (2020). Decreased inhibitory control after partial sleep deprivation in individuals reporting binge eating: preliminary findings. PeerJ 8:e9252. doi: 10.7717/peerj. 9252

Chaput, J. P., Yau, J., Rao, D. P., and Morin, C. M. (2018). Prevalence of insomnia for Canadians aged 6 to 79. Health Rep. 29, 16-20.

Cohen, J. (1992). A power primer. Psychol. Bull. 112, 155-159.

Connolly, S. L., Wagner, C. A., Shapero, B. G., Pendergast, L. L., Abramson, L. Y., and Alloy, L. B. (2014). Rumination prospectively predicts executive functioning impairments in adolescents. J Behav. Ther. Exp. Psychiatry 45, 46-56. doi: 10.1016/j.jbtep.2013.07.009

Couyoumdjian, A., Sdoia, S., Tempesta, D., Curcio, G., and Rastellini, E. (2010). The effects of sleep and sleep deprivation on task-switching performance. J. Sleep Res. 19, 64-70. doi: 10.1111/j.1365-2869.2009. 00774.x

Ehring, T., Zetsche, U., Weidacker, K., Wahl, K., Schonfeld, S., and Ehlers, A. (2011). The perseverative thinking questionnaire (PTQ): validation of a content-independent measure of repetitive negative thinking. J Behav. Ther. Exp. Psychiatry 42, 211-232.

Hartley, S., Bucci, S., and Morrison, A. P. (2017). Rumination and psychosis: an experimental, analogue study of the role of perseverative thought processes in voice-hearing. Psychosis 9, 184-186. doi: 10.1080/17522439.2017.12 80073

Hartmann, E., Rey-Mermet, A., and Gade, M. (2019). Same same but different? Modeling N-1 switch cost and N-2 repetition cost T with the diffusion model and the linear ballistic accumulator model. Acta. Psychol. 198:102858. doi: 10.1016/j.actpsy.2019.05.010

Harvey, A. G. (2008). Insomnia, psychiatric disorders, and the transdiagnostic perspective. Curr. Dir. Psychol. Sci. 17, 299-303. doi: 10.1111/j.1467-8721.2008. 00594.x
Hayes, A. F. (2013). Introduction to Mediation, Moderation, and Conditional Process Analysis: A Regression-Based Approach. New York, NY: The Guilford Press.

Hertenstein, E., Feige, B., Gmeiner, T., Kienzler, C., Spiegelhalder, K., Johann, A., et al. (2019). Insomnia as a predictor of mental disorders: a systematic review and meta-analysis. Sleep Med. Rev. 56, 98-103.

Hilt, L. M., Leitzke, B. T., and Pollak, S. D. (2014). Cognitive control and rumination in youth: the importance of emotion. J. Exp. Psychopathol. 5, 302-313. doi: 10.5127/jep.038113

Jiang, X. I., Zheng, X. Y., Yang, J., Ye, C. P., Chen, Y. Y., Zhang, Z. G., et al. (2015). A systematic review of studies on the prevalence of Insomnia in university students. Public Health 129, 1579-1584. doi: 10.1016/j.puhe.2015. 07.030

Lancee, J., Eisma, M. C., van Zanten, K. B., and Topper, M. (2017). When thinking impairs sleep: trait, daytime and nighttime repetitive thinking in insomnia. Behav. Sleep Med. 15, 53-69. doi: 10.1080/15402002.2015.10 83022

Law, K. C., and Tucker, R. P. (2018). Repetitive negative thinking and suicide: a burgeoning literature with need for further exploration. Curr. Opin. Psychol. 22, 68-72. doi: 10.1016/j.copsyc.2017.08.027

Marchetti, I., Mor, N., Chiorri, C., and Koster, E. H. W. (2019). The brief state rumination inventory (BSRI): validation and psychometric evaluation. Cogn. Ther. Res. 42, 447-460. doi: 10.1007/s10608-01 8-9901-1

Morin, C. M., Belleville, G., Bélanger, L., and Ivers, H. (2011). The insomnia severity index: psychometric indicators to detect insomnia cases and to evaluate treatment response. Sleep 34, 601-608. doi: 10.1093/sleep/34. 5.601

Nolen-Hoeksema, S., Wisco, B. E., and Lyubomirsky, S. (2008). Rethinking rumination. Perspect. Psychol. Sci. 3, 400-424. doi: 10.1111/j.1745-6924.2008. 00088.x

Ottaviani, C., Thayer, J. F., Verkuil, B., Lonigro, A., Medea, B., Couyoumdjian, A., et al. (2016). Physiological concomitants of perseverative cognition: a systematic review and meta-analysis. Psychol. Bull. 142, 231-259. doi: 10.1037/bul00 00036

Philipp, A. M., and Koch, I. (2006). Task inhibition and task repetition in task switching. Eur. J. Cogn. Psychol. 18, 624-639. doi: 10.1080/09541440500 423269

Raes, F. (2012). Repetitive negative thinking predicts depressed mood at 3-year follow-up in students. Psychophysiology 34, 497-501. doi: 10.1007/s10862-0129295-4

Ruscio, A., Seitchik, A. E., Gentes, E. L., Jones, J. D., and Hallion, L. S. (2011). Perseverative thought: a robust predictor of response to emotional challenge in generalized anxiety disorder and major depressive disorder. Behav. Res. Ther. 49, 867-874. doi: 10.1016/j.brat.2011.10.001

Sdoia, S., and Ferlazzo, F. (2008). Stimulus-related inhibition of task set during task switching. Exp. Psychol. 55, 322-327. doi: 10.1027/1618-3169.55. 5.322

Snyder, H. R., Miyake, A., and Hankin, B. L. (2015). Advancing understanding of executive function impairments and psychopathology: bridging the gap between clinical and cognitive approaches. Front. Psychol. 6:328. doi: 10.3389/ fpsyg.2019.00328

Trick, L., Watkins, E., Windeatt, S., and Dickens, C. (2016). The association of perseverative negative thinking with depression, anxiety and emotional distress in people with long term conditions: a systematic review. J. Psychosom. Res. 91, 89-101. doi: 10.1016/j.jpsychores.2016.11.004

van der Zeerde, T., van Straten, A., Effting, M., Kyle, S. D., and Lancee, L. (2019). Does online insomnia treatment reduce depressive symptoms? A randomized controlled trial in individuals with both insomnia and depressive symptoms. Psychol. Med. 49, 501-509. doi: 10.1017/s00332917180 01149

Wahl, K., Ehring, T., Kley, H., Lieb, R., Meyer, A., Kordon, A., et al. (2019). Is repetitive negative thinking a transdiagnostic process? A comparison of key processes of RNT in depression, generalized anxiety disorder, obsessivecompulsive disorder, and community controls. J. Behav. Ther. Exp. Psychiatry 64, 45-63.

Whitmer, A. J., and Banich, M. T. (2007). Deficits in different forms of rumination. Psychol. Sci. 18, 546-553. doi: 10.1111/j.1467-9280.2007.01936.x 
Whitmer, A. J., and Gotlib, I. H. (2012). Switching and backward inhibition in major depressive disorder: The role of rumination. J. Abnorm. Psychol. 121, 570-578. doi: 10.1037/a0027474

Yang, Y., Cao, S., Shields, G. S., Teng, Z., and Liu, Y. (2016). The relationships between rumination and core executive functions: a meta-analysis. Depress. Anxiety 34, 37-50. doi: 10.1002/da. 22539

Zoccola, P. M., Dickerson, S. S., and Sumam, L. (2009). Rumination predicts longer sleep onset latency after an acute psychosocial stressor. Psychosom. Med. 71, 771-775. doi: 10.1097/psy.0b013e3181 ae 58 e 8
Conflict of Interest: The authors declare that the research was conducted in the absence of any commercial or financial relationships that could be construed as a potential conflict of interest.

Copyright (c) 2020 Ballesio, Cerolini, Vacca, Lucidi and Lombardo. This is an openaccess article distributed under the terms of the Creative Commons Attribution License (CC BY). The use, distribution or reproduction in other forums is permitted, provided the original author(s) and the copyright owner(s) are credited and that the original publication in this journal is cited, in accordance with accepted academic practice. No use, distribution or reproduction is permitted which does not comply with these terms. 